\title{
Dairy donkey: an alternative building layout
}

\author{
Carlo Bibbiani, ${ }^{1}$ Patrizio Biagini, ${ }^{2}$ Federica Salari, ${ }^{1}$ Mina Martini ${ }^{1}$ \\ ${ }^{1}$ Department of Veterinary Science, University of Pisa; ${ }^{2}$ Bandite di Scarlino, Scarlino (GR), Italy
}

\begin{abstract}
In Italy, donkey dairies are growing in popularity to produce an alternative milk source for human infants. The use of donkey milk is not limited to the paediatric field, being used in geriatric nutrition and in the cosmetic industry. In 2010, the Department of Veterinary Science, University of Pisa, was involved in a project supported by the Tuscany Region Rural Development Fund 20072013 dealing with the creation of a donkey milk productive chain. The project has as its main partner the Bandite di Scarlino, an agro-forestry complex of considerable size. It covers approximately 9000 hectares, which fall in Scarlino, Castiglione della Pescaia, Follonica and Gavorrano municipalities, under Tuscany regional control. The so-called FILAMI project (Filiera Latte Asino Amiatino, acronym of the goal of the project in Italian) had among its goals helping preserve an indigenous breed of donkeys called Amiatina. Salimei (2011) assessed that the presence of the foal during milking does not affect milk ejection in jennies (the female donkey) adapted to the milking procedure, but when foals are not present the milking routine is more manageable for the animals and for optimal milk harvest; in accordance, in the present farming system the jenny has been kept separately from the foal during the milking session. To this purpose, the stable, the milking parlour and the paddocks must be well organised with a proper milking routine. Milking facilities must be designed for the milk treatment and storage at low temperature or for freezing. Currently, the milk undergoes a pasteurisation cycle for human consumption, or directed to the cosmetic industry. Moreover, the milking facilities should fulfil the creation of a milk collection centre in order to play an important role between the dairy donkey farms and the dairy industry.
\end{abstract}

Correspondence: Carlo Bibbiani, Department of Veterinary Science, University of Pisa, viale delle Piagge 2, 56124 Pisa, Italy. Tel.: +39.050.2216813 - Fax: +39.050.2216706.

E-mail: carlo.bibbiani@unipi.it

Key words: Dairy donkey; farm building design; milking facilities.

Received for publication: 22 November 2016.

Accepted for publication: 9 February 2017.

CC Copyright C. Bibbiani et al., 2017

Licensee PAGEPress, Italy

Journal of Agricultural Engineering 2017; XLVIII(s1):637

doi:10.4081/jae.2017.637

This article is distributed under the terms of the Creative Commons Attribution Noncommercial License (by-nc 4.0) which permits any noncommercial use, distribution, and reproduction in any medium, provided the original author(s) and source are credited.
In this paper, the technical and technological aspects of the donkey stable, paddocks, milking parlour, mobile milking unit, and processing plants have been described and the main issues addressed.

\section{Introduction}

The dairy donkey enterprise can be classified as innovative, even if the donkey milk has a well-documented utilisation since thousands of years ago, and many dairy donkey farms started up during the last years mainly in Italy, France, and Spain, while China, Ethiopia and Pakistan have been raising donkeys for centuries (Salimei, 2011). Milk production is very different between breeds. European Community legislation defines specific health requirements for the primary production of raw milk (Salimei, 2011). Recently, the Department of Veterinary Science, University of Pisa, was involved in a project supported by the Tuscany Region Rural Development Fund 2007-2013 (PSR 2007-2013, Misura 124, Cooperation for development of new products, processes and technologies in the agriculture, food, and forestry sector, aiming to create a Dairy Donkey productive chain for human consumption, and for cosmetics. In Italy, donkey dairies are growing in popularity to produce an alternative milk source for human infants (Martini et al., 2014). Moreover, the Department has been commissioned to design the new facilities for donkey's milking. The project involves as main partners the Bandite di Scarlino, an agro-forestry complex of considerable size, approximately 9000 hectares, within Scarlino, Castiglione della Pescaia, Follonica and Gavorrano municipalities, under Tuscany regional control, and the Istituto Zooprofilattico Sperimentale delle Regioni Lazio e Toscana, along with a famous local dairy industry, a consortium of milk producers (Consorzio Produttori Latte Maremma), and other associations.

The goal of the project is to create a sustainable chain of ass's milk from an environmental, economic, and social point of view. In suited agricultural areas, the donkey's milk can be an innovative productive choice for the farms needing to identify new market opportunities (multi-functionality), as traditional vocations do not allow for adequate integration of agricultural income.

At the same time, it helps preserving indigenous breed of donkeys, in this case called Amiatino, as a protection of biodiversity. The native donkey breed Amiatino (or Amiata) derived its name from the traditional farming area of Mount Amiata (Tuscany, Italy). The population was once reared as working animals, but agricultural industrialisation brought the population near to extinction. Only recently measures to safeguard the population have been introduced, and the Amiatino donkey is now used for donkey rides, pet therapy, and also for milk production, showing a good attitude to this task.

As general goals, the organisation of the chain of donkey's milk would allow: i) the implementation of appropriate health 
management programs and of reproductive activity; ii) the development of specific feed schedule for milk production asses in relation to farm feed availability; iii) the definition of a farm protocol for the production of milk that meets the requirements, depending on the intended use; iv) the creation of a network of dairy donkey farmers; v) establishing a quality milk production, aimed at upgrading farms as donkey milk suppliers; vi) the creation of a milk collection centre in order to play an important role between the dairy farms and the dairy industry; vii) the rationalisation of the production on the territory that could establish a delivery chain and make prices more accessible to the consumer. Before the beginning of the FILAMI project, no donkeys were reared in the site: at the moment, there are about 140 jennies reared outdoors, 40 of whom are routinely machine milked. The present paper describes task vi), that is to say the identification and the proposal of a new building scheme of the milking facilities. Moreover, under the goals of tasks iv) and vii), it deals with the mobile milking unit (MoMU) description. Finally, a comparison test between milking parlour and MoMU has been reported.

A

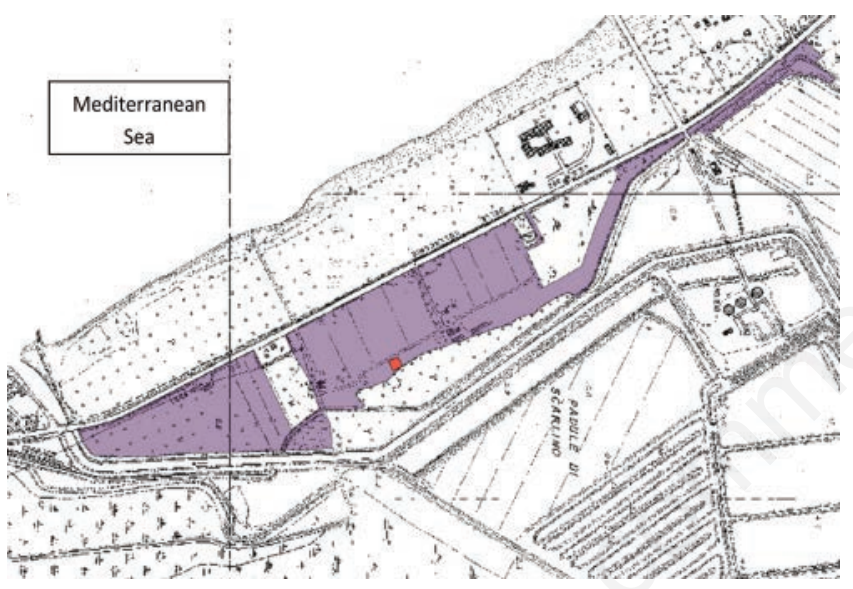

B

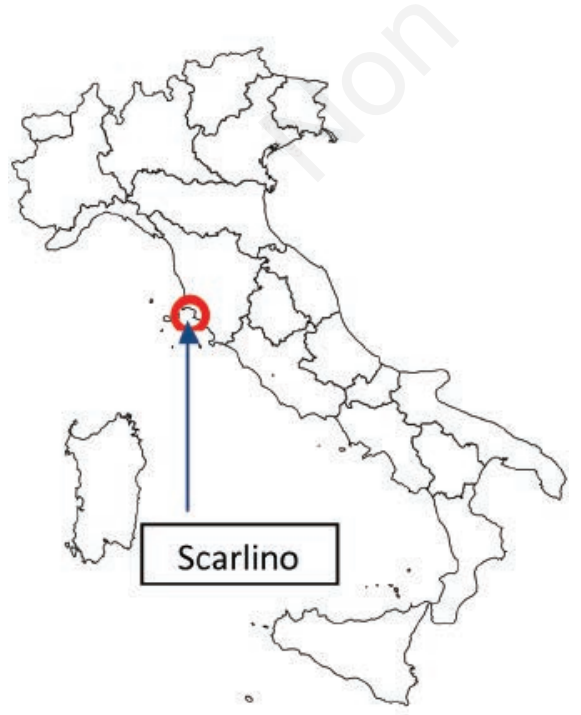

Figure 1. A) Location of the paddock (purple) and new donkey milking parlour and milk centre (red); B) Scarlino municipality in Italy (red circle).

\section{Materials and methods}

\section{The functional relationships among components}

Quoting what Josi Flaba, member of the CIGR Section II Working Group No. 14 Cattle Housing, wrote: the definition of dairy (cattle) housing characteristics requires the understanding and knowledge of various interrelated subjects such as: ethology, animal husbandry, handling of the animals, optimisation of the working conditions, protection of the people against accidents, ventilation of livestock buildings, properties of materials, landscape integration, manure management, etc. (CIGR, 2014), gives us basic information on what designing farm buildings consists.

The systematic layout planning (SLP) methodology developed for the planning of industrial facilities, can be applied for the generation of optimal layouts in livestock farms (Pérez et al., 2004; Tomaselli et al., 2004), and as far as it concerns, in dairy donkeys facilities. The SLP methodology has a six phases: i) definition: the problem is defined and an analysis of the type and quantity of products that will experience flows in the layout is carried out; ii) analysis: the relational diagram of routes and/or activities is obtained and interrelated with the space required for each activity and the available space, thus obtaining the relational diagram of spaces; iii) synthesis: the generation of alternative layouts is carried out; iv) evaluation: each alternative is studied in detail; v) selection: the best alternative is chosen; vi) implementation and pursuit: the selected installation is carried out (Pérez et al., 2004).

Although there are a wide variety of SLP problems, the objective is the same, which is to increase the efficiency of the systems. From the SLP viewpoint, the efficiency of a system can be increased from several aspects, such as the material handling cost, the adjacency relationships (Wascher and Merker, 1997), the personnel flow, the aesthetic value, etc. (Shuai, 2013). SLP approach explicitly addresses these issues making them criteria for evaluating the layout plans.

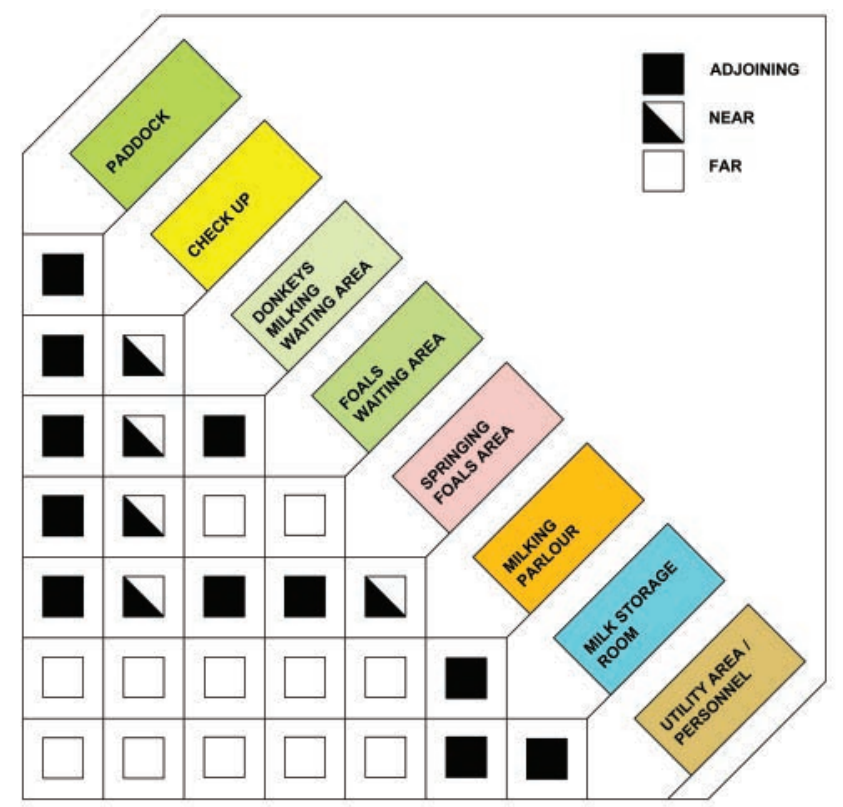

Figure 2. Correlation matrix among functional areas. 
In the present design, the SLP has been carried out applying the adjacency relationships, and the donkeys/personnel flow. The donkey is a rustic animal and, as such, there are low technological requests regarding the farm buildings. The major components around which a milking centre is planned are the paddock, the milking waiting area, the milking parlour, the milk storage room, and facility for health surveillance (check up), designed to respect the relationship of adjoining, near and far position, and to optimise workers and donkeys comfort and traffic patterns.
The milking parlour is the special area where jennies are brought to be milked and then returned to their housing facility or to paddock. An elevated parlour, with donkeys standing in line one or two sides facing the operator doing the milking, is the best choice for this species.

The waiting area is a holding pen required for confining jennies prior to their entrance into the milking parlour, and it is connected with the paddock through paths allowing the separation of foals from donkeys. Separation is compulsory to prevent foals

C
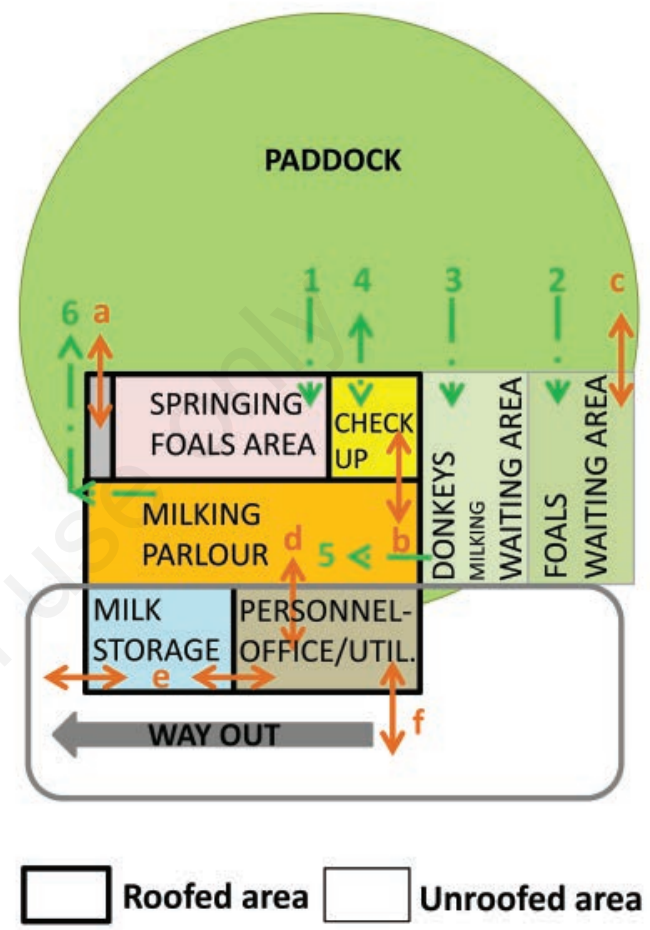

D

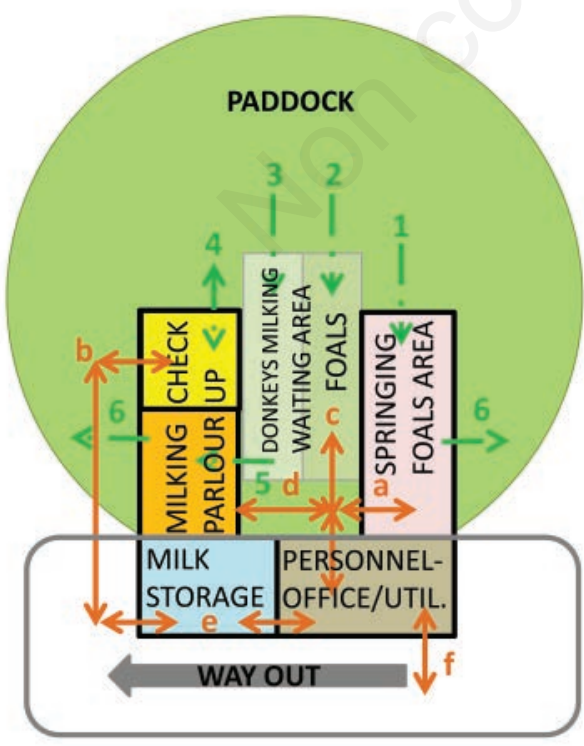
Roofed area Unroofed area
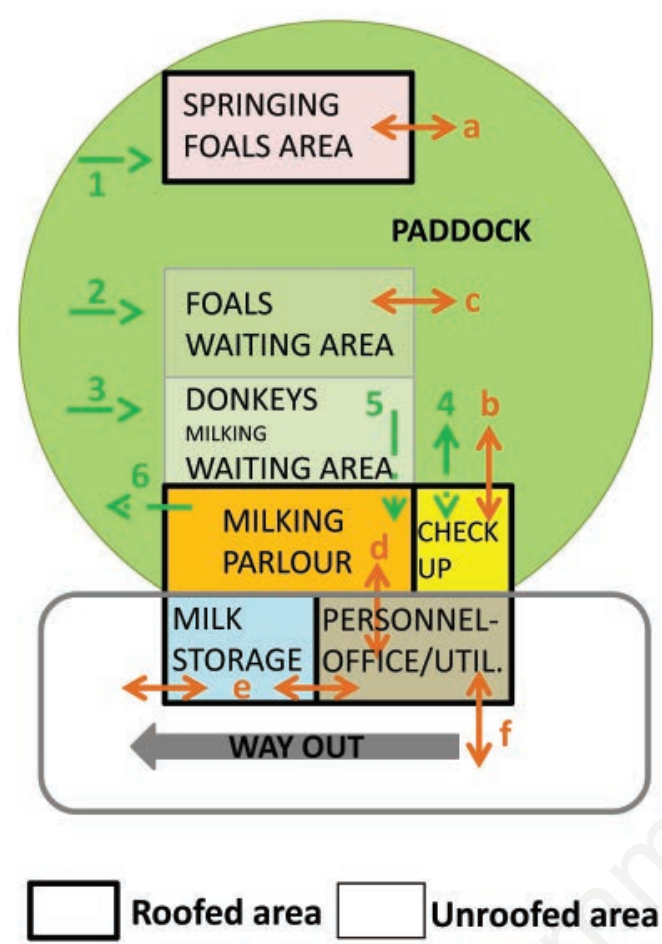

B

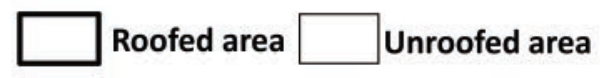

Connections for employee flows

a Birth assistance - Straw - Waste

b Health Check up- Treatments

c Feeding - Waste removal

d Milking

e Milk Packaging \& Delivering

f Personnel facilities /Utility

From paddock

1 Springing foals - Maternity

2 Foals waiting area

3 Donkeys milking waiting area

4 Check up

From donkeys waiting area

5 Milking parlour

To

6 Paddock
Connections for animal flows

Figure 3. A) Split layout; B) U-shape layout; C) compact layout; D) legend. 
sucking milk. In fact, foals must be separated at least three hours before milking the jennies, given the morphological type of the udder and its low storage capability.

The paddock area takes into account the rationalisation of patterns and the installation of specific equipment (sheds, troughs, feeders, etc.). Occasionally the donkeys undergo health surveillance in check up boxes. Moreover, a springing foals area could be useful, to cope with some difficulties or problems before the birth event. Generally, all other births take place outdoor at Bandite di Scarlino farm.

The milk storage room is devoted to milk cooling and storage. It has facilities for clean up and sanitation of milking equipment. In short, milking and storage at $4{ }^{\circ} \mathrm{C}$ must be carried out in clean places, designed and equipped to avoid the risk of contamination (European Parliament and the Council, 2004). Personnel facilities in workplaces must comply with the requirements of protection of the people against accidents (Italian Regulation, 1994), and of sanitary installations such as toilets and washrooms and facilities for storing clothes. Thus, a different connection for personnel and for product inlet/outlet must be provided.

The utility room houses the mechanical equipment necessary for cleaning, milking, and cooling of milk such as vacuum pumps, refrigeration compressors, and hot water heater.

In order to fulfil the creation of a network of dairy donkey farmers, aimed at upgrading small farms as donkey milk suppliers, a complete mobile milking unit has been designed. MoMU should be lightweight, compact, extremely portable, and designed perfectly for farm, and field use. MoMU must provide an efficient and convenient way of milking animals in the field, or in the barn, or when attending agricultural shows, for small size herd or animals in remote pens. Pathways for animals and workers among the different components help to define a flow table (Pérez et al., 2004), taking into account the movement of animals, people, machinery, raw materials (fodder, concentrates, etc.), products (milk) and byproducts (manure). To describe the functional relationship among the components a correlation matrix must be sketched (Tomaselli et al., 2004). This kind of matrices is an input parameter of computer programs for layout generation based on genetic algorithms (Pérez et al., 2002), or can be exploited by engineers to design different layouts under established hypotheses.

\section{Evaluation and comparison of milk production}

Milk production was evaluated at the morning milking sorting out 8 pluriparous jennies in two periods during lactation (about 90 and 120 days of milk) in spring and summer. Other jennies were in different phases of lactation and for this reason were not enrolled in the comparison. Jennies were between 6 and 10 years and weighted about 250-300 kg. They were reared outdoor and fed with about $2.5 \mathrm{~kg} \mathrm{day}^{-1}$ head $^{-1}$ of concentrate for dairy donkeys and polyphite hay ad libitum.

Experiment lasted 4 days for each lactation period during which jennies were milked alternatively using milking parlour (32 samples) and the MoMU (32 samples). Routinely, foals were physically separated from the jennies $3.5 \mathrm{~h}$ before the first milking, following Martini et al. (2015), and Salimei and Fantuz (2012). The foals were kept separated until the end of second milking, for a total of $8 \mathrm{~h}$. During the separation period the foals were fed with with about $0.5 \mathrm{~kg} \mathrm{day}^{-1}$ head $^{-1}$ of concentrate for dairy donkeys and polyphite hay ad libitum. All the animals had free water access.

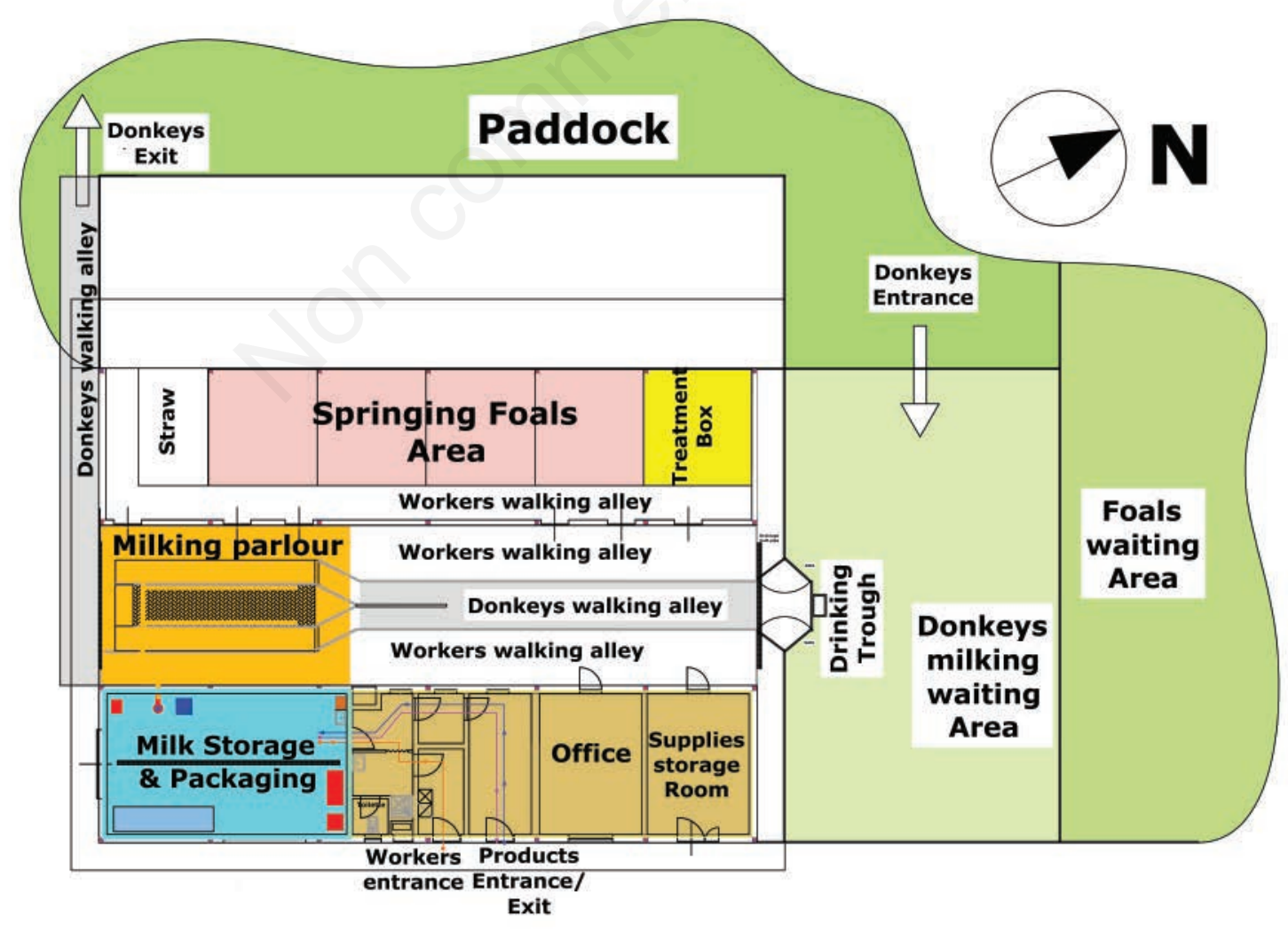

Figure 4. Layout of the solution developed at Scarlino (areas are shadowed by the same colours as components showed in Figure 2 and 3 ). 


\section{Statistical analysis}

Milk production data were statistically analysed by ANOVA test $\left(\mathrm{JMP}^{\circledR}\right.$ release 7 - 2007, SAS Institute Inc., Cary, NC, USA), and the model included the milking system and its interaction with animal as fixed effects, and animal as random effect.

\section{Results and discussion}

\section{The FILAMI project at Scarlino}

The FILAMI project has been developed at Scarlino (Figure $1 \mathrm{~A}$ and $\mathrm{B}$ ) where the Bandite di Scarlino run a multi-purpose farm, but no donkeys at all were reared before. The project thrust the construction of the donkey facility, paddocks, milking parlour, mobile milking unit, milk storage room and milk collection centre.

Following the SLP methodology, a correlation matrix among components has been plot (Figure 2). This matrix has been originated by two clusters: the workers area and the animal area linked by the milking parlour. Due to the goals of the milk collection centre, the milk storage must be accessible to many farmers. Other components can be joined combining different purposes.

Under these assumptions, three alternative layouts have been sketched up (Figure 3). Figure 3A shows a split solution, with the main building fulfilling all the requirements but the springing foals area (Figure 3B) sketches a layout with all the functional areas adjoined together with an U-shape form, for example protecting from wind adversity. Figure $3 \mathrm{C}$ shows a compact solution with the shortest roofed paths for employees.

Layout $3 \mathrm{C}$ has been chosen as the most convenient for the farm requirements giving birth to the plot shown in Figure 4.

\section{Milking area and mobile milking unit}

In the donkeys milking waiting area rather short requirements are needed, mostly free water and shade shelters, if any. Prior to milking the whole group enters into a waiting area, approximately $150 \mathrm{~m}^{2}$ large (Figure 5A), equipped with a gate to drive the jennies toward the parlour. The path toward the milking parlour has an unexpected length due to the presence of other components in line, to the location of the milking pit and milk the storage room toward the farm entrance. A primary reason for a milking parlour is to increase the number of donkeys milked per person per hour. Other motivating factors include attracting and keeping hired or family labour. These benefits must be weighed against the capital cost of the parlour. The equipment for milking can be very simple, such as a milking trolley suitably adapted from sheep and goats type, or thoroughly organised, such as a milking parlour. The chosen layout has an elevated parlour with six stalls, three each side, on a concrete pad (Figure 6A and B).

Donkey standing positions are made of zinc-coated steel; they allow the individual fixation and positioning of animals for comfortable milking. Each stall measures $190 \mathrm{~cm}$ in length, $60 \mathrm{~cm}$ in width (Figure 6C and D). Here all the jennies to be milked are driven by one worker and fed with food concentrate in a trough. Milking of one donkey after the other proceeds with modest pauses mainly for moving the milking cluster from one animal to the next (Failla, 2008). Milking operations are quite quick, being the Time per Turn around $6 \mathrm{~min}$, and Turns per Hour equal to 10. In dairy jennies, average milk ejection varies from 40 to 90 s per milking and is directly related to the amount of milk harvested, in accordance with literature (Salimei, 2011). Thanks to this arrangement, 40 jennies are routinely machine milked in $40 \mathrm{~min}$.
Vacuum system provides the stabile vacuum level of $46 \pm 1 \mathrm{kPa}$ in milking mode and consists of 1" diameter stainless steel, and liquid-packed ring vacuum station (up to six clusters driven). Adjustment and keeping of vacuum-gauge pressure into vacuum and milk pipelines perform by means of vacuum-regulator (by Ima Grosseto ${ }^{\circledR}$ s.r.1.).

Milk intake system consists of 30-L milk tank. Milk intake unit is completed with one-milk pump. The unit is equipped with a pneumatic pulsator and provides the automatic stimulation of udder under a 50:50 pulsation ratio, and a pulsation rate of 120 cycles min $^{-1}$ (Salimei, 2011; ISO, 2007). The control unit operates in half-automatic mode with the switching off and removing of milking apparatus at an operator's command. The milk pipeline is made of stainless steel with polished internal surface and the minimal diameter of pipes is 1 " $\times 1.5 \mathrm{~mm}$. Milk is pumped to the milk storage room where is filtered and pasteurised (Evopastò $30,4 \mathrm{~kW}$, V 400 3-phase, by Telme ${ }^{\circledR}$ ), warming it up to temperature of $65^{\circ} \mathrm{C}$ for about 30 minute, and then refrigerated at $4^{\circ} \mathrm{C}$, to keep it hygienic (Figure 7A and B).

Once the refrigeration process is complete, it undergoes packaging: it is bottled into 0.75 litre bottles and placed in a refrigerator at $4^{\circ} \mathrm{C}$, for human consumption, or poured into 3-L's bags and frozen at $-18^{\circ} \mathrm{C}$, if destined to cosmetics (freezer by Coldline ${ }^{\circledR}$, adjustable temperature range $-10^{\circ} \div-30^{\circ} \mathrm{C}$, volume 700 litre, 1.18 $\mathrm{kW}$ power, refrigerant $\mathrm{R} 404 \mathrm{a})$.

All the system is then automatically washed. The automat has multistage washing and possibility of the precise programming of all processes: quantity of water, concentration of cleaning solutions, heating temperature and washing time of the unit. The unit has alkaline and acid cleaning agents. An efficient wash up routine will help maintain milk quality, and prolong the life of milking equipment.

The milk storage room has been south oriented, due to connection with the farm entrance. To cope with over-irradiation, a thick insulation has been built in the walls, and a thorough climatic control has been provided, completely powered by photovoltaics panels located on the roof. The room has a second purpose: the creation of a milk collection centre in order to help small dairy farms starting this activity. To this extent, a separated storage tank has been provided for keeping milk prior its process into the pasteuriser. The mobile milking system has been designed and built (Figure 7C). MoMU is capable of milking two jennies at a time (Figure 7D) with a bucket milking machine, that is a milking machine in which milk flows from two clusters into a portable milk-receiving bucket ( 25 $\mathrm{L}$ ), connected to the vacuum system by $1 / 2$ " vacuum hose, and a $0.75 \mathrm{~kW}$ oil free, direct drive vacuum pump with silencer (by Ima Grosseto ${ }^{\circledR}$ s.r.1.). This machine has the same parameters set-up as the above mentioned parlour system machine. Comes with the MoMU, complete with a stand-alone power unit, a wash up system, a refrigerator, and several small stainless steel storing tanks (3, 5 or $10 \mathrm{~L})$, one each farm to be visited.

\section{Other components}

Beside the milking area following the request of the donkey breeder, maternity and springing foals area has been designed (Figure 5B), which is $400 \mathrm{~cm}$ in length, $420 \mathrm{~cm}$ in width each box, in order to allow proper assistance to the delivering donkey. A straw bedding is the best choice, with a straw renovation of at least $5 \mathrm{~kg} \mathrm{day}^{-1}$ box $^{-1}$. Close to this area, there is one treatment box that is specifically addressed for all types of donkeys care for which the donkey needs to stand still, for insemination, administration of medication, and hoof care. It is not an isolation box and for this 

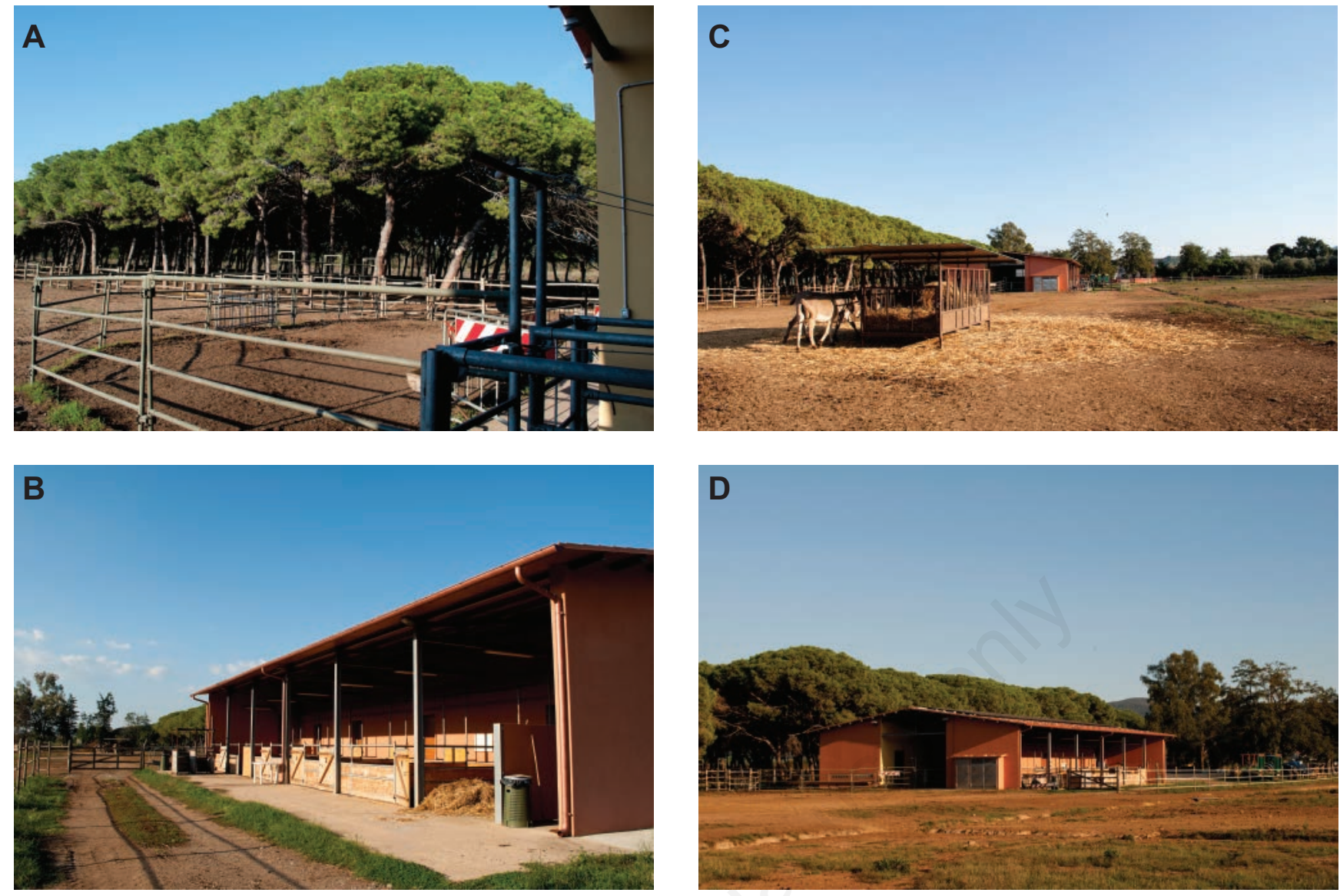

Figure 5. A) Milking waiting area; B) maternity and springing foal area; C) hay feeder; D) paddock.
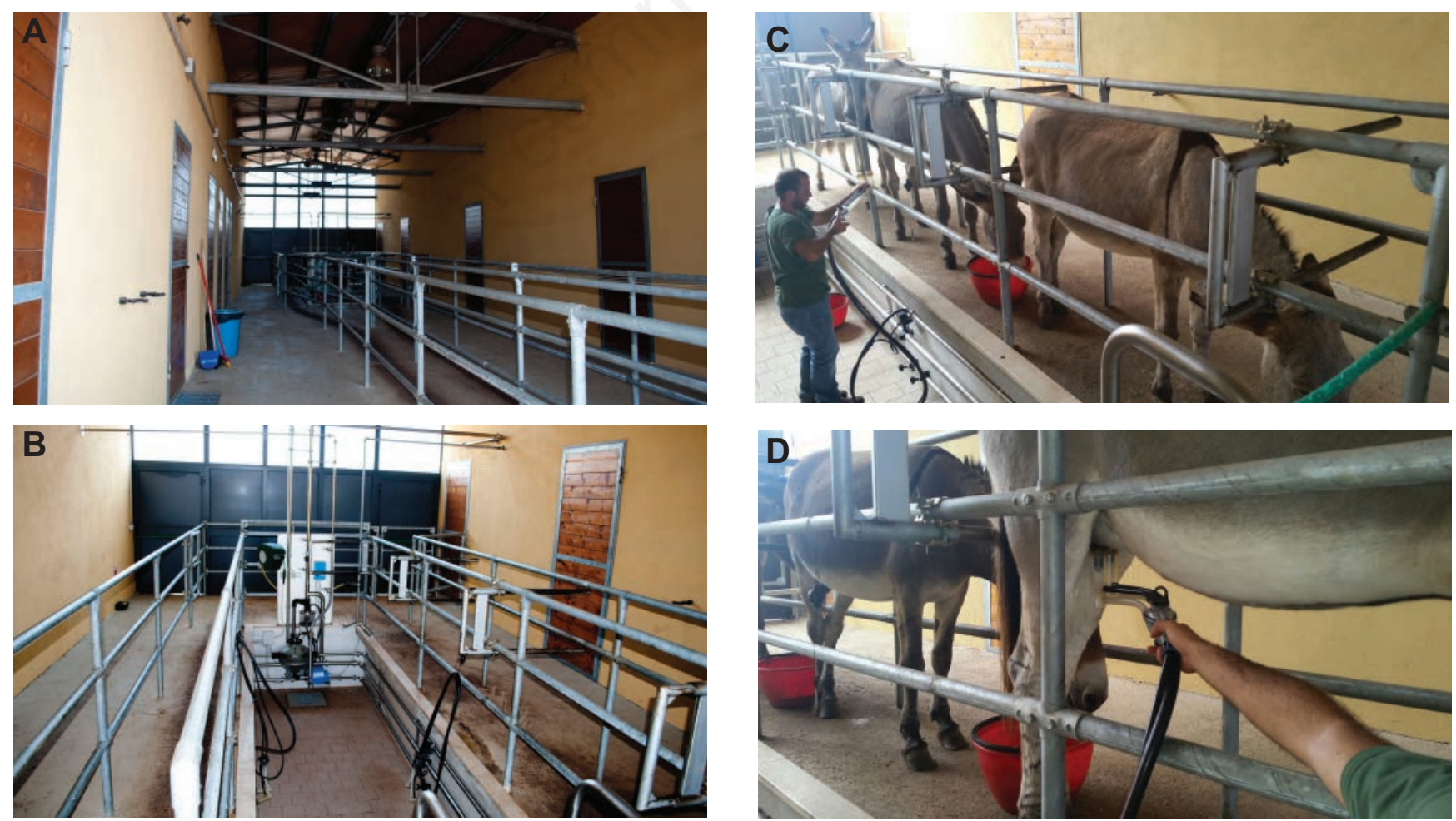

Figure 6. A) Workers and donkeys alleys; B) milking parlour and pit; C) milking donkeys at stalls; D) milking unit. 
reason it can be located nearby springing foals. It is better be located in proximity to the milking facilities in order to drive easily donkeys before/after milking. Paddock and feeding facilities complete the farm with sheds, troughs and feeders (Figure 5C and D).

\section{Evaluation of milk production under different milking system}

In Table 1 the average production of each milked jenny are reported along with the two different milking system and their significance. Milking lasted until the operator detected very low milk flow. Data show that milking system has a significant influence on milk production, with means equal to $532.8 \pm 219.1 \mathrm{~mL} \mathrm{milking}^{-1}$ and $349.1 \pm 132.6 \mathrm{~mL}$ milking ${ }^{-1}$ (mean \pm standard deviation; $\mathrm{P}<0.01$ ) respectively for the milking parlour and MoMU. According to Salimei (2011), in different experimental conditions, the observed average milk yield of some Italian breeds (Martina Franca, Ragusano, Romagnolo, and Sardo) ranges from 350 to 850 $\mathrm{mL}$ per milking. This variability is a result of many factors such as management of foals and dams, individual milkability, milking procedure, stage of lactation, body size and condition, and feeding. Donkeys are rustic animals accustomed to climatic adversity; as such, the microclimate is not reported as a main factor affecting milk yield. Anyhow, during the two periods in which the test was

Table 1. Average production $(\mathrm{mL})$ of each milked jenny along with the two different milking system and their significance.

\begin{tabular}{lccc} 
Animal & Mean production at milking parlour $(\mathrm{mL})$ & Mean production at MoNU (mL) & P value \\
1 & $699.8^{\mathrm{a}}$ & $434.3^{\mathrm{b}}$ & 0.039 \\
2 & 336.4 & 416.9 & 0.506 \\
\hline 3 & 653.8 & 457.8 & 0.117 \\
4 & $499.3^{\mathrm{a}}$ & $208.8^{\mathrm{b}}$ & 0.026 \\
\hline 5 & 447.2 & 469.7 & 0.852 \\
6 & 414.2 & 317.7 & 0.426 \\
\hline 7 & 388.9 & 288.4 & 0.408 \\
\hline
\end{tabular}

MoMU, mobile milking unit. a,bignificant difference between values.
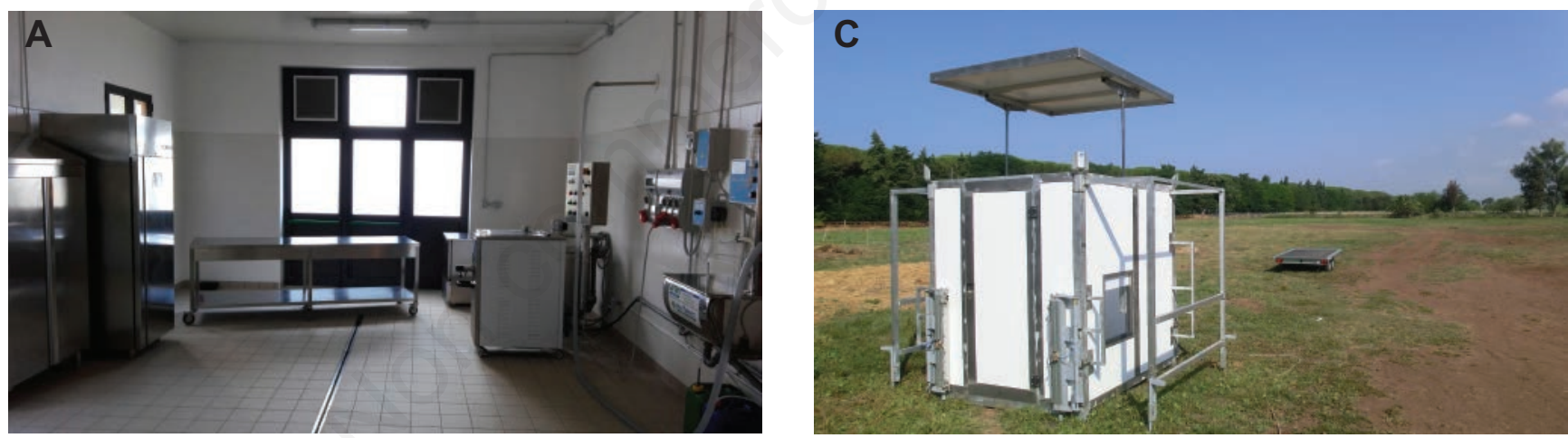

B
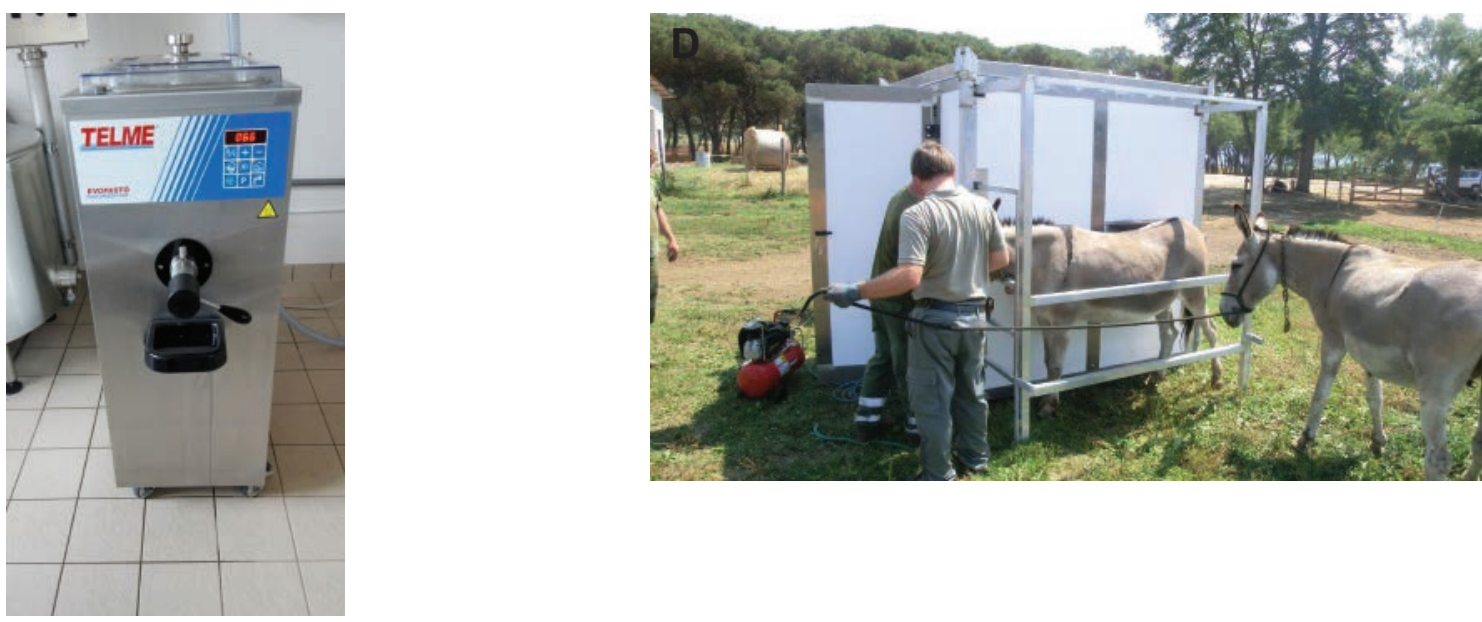

Figure 7. A) Milk cooling and storage; B) pasteuriser (Telme, Evopastò 30); C) MoMU and carriage; D) MoMU and milking donkeys. 
run, the climatic conditions remained comparable each day with the following, and thus had a negligible effect on the test.

Evaluating the differences over each jenny, only 3 animals out of 8 show a significant value (Table 1), suggesting that the different mobile milking system can perform as well as the milking parlour if only enough training would have been allowed. Doreau (1991) also stated that management of dairy equids could influence the amount of milk extracted by the mammary gland since it is characterised by low capacity. In our study, all the jennies were routinely milked at the milking parlour and thus the MoMU can be seen as a divergence from what the animals are accustom to; anyhow, according to Salimei and Chiofalo (2006), asses always show a quick adaptation to the dairy management routines.

\section{Conclusions}

In order to create a Donkey Milk productive chain, the Tuscany Region Rural Development Fund 2007-2013 financed a project, called FILAMI project (Filiera Latte Asino Amiatino), at Scarlino, where the Bandite di Scarlino, an agro-forestry complex under Tuscany regional control, run a multi-purpose farm. The project thrust the construction of the donkey stable, paddocks, milking parlour, milk storage room and milk collection centre, beside other components. A MoMU was also built in order to help the creation of a network of dairy donkey farmers, upgrading farms as donkey milk suppliers; to this extent, the milk collection centre should play an important role between the dairy farms and the dairy industry.

The MoMU was tested versus the milking parlour to evaluate the differences in milk production: the comparison showed that there is a significant difference in favour of the milking parlour probably due to the adaptation of jennies to the milking parlour routine.

Up to now, the dairy activity has been started, there are 140 jennies, and 40 of them are routinely milked. All the design method and the chosen components of the dairy donkey farm have been described.

\section{References}

CIGR, 2014. The design of dairy cow and replacement heifer housing. Report of the CIGR Section II Working Group $\mathrm{N}^{\circ} 14$ Cattle Housing. Commission Internationale du Génie Rural [International Commission of Agricultural and Biosystems Engineering]. Available from: http://www.cigr.org/documents/Design_of_dairy_cow_and_remplacement_heifer_hous ing-CIGR_working_group_Cattle_housing-2015.pdf

Doreau M. 1991. Le lait de jument. INRA Prod. Anim. 4:297-302.

European Parliament and the Council, 2004. Regulation (EC) No $853 / 2004$ laying down specific hygiene rules for on the hygiene of foodstuffs. In: Official Journal No. 139, 30/4/2004, pp 55-205.
Failla S. 2008. Assessment of work organisation and analysis of energy consumption in mechanical milking and donkey milk processing. pp 1-7 in International Conference "Innovation Technology to Empower Safety, Health and Welfare in Agriculture and Agro-food Systems", Ragusa, Italy.

ISO, 2007. Milking machine installations - Vocabulary. Norm ISO 3918:2007. International Organisation for Standardisation Publ., Geneva, Switzerland.

Italian Regulation, 1994. Application of the Council Directive (EEC) No. 89/391, 89/654, 89/655, 89/656, 90/269, 90/270, 90/394 and 90/679 riguardanti il miglioramento della sicurezza e della salute dei lavoratori sul luogo di lavoro. LD 626/1994. In: G.U. No. 265, 12/11/1994.

Martini M., Altomonte I., Manica M., Salari F. 2015. Changes in donkey milk lipids in relation to season and lactation. J. Food Compos. Analysis 41:30-4.

Martini M., Altomonte I., Salari F., Caroli A.M. 2014. Monitoring nutritional quality of Amiata donkey milk: Effects of lactation and productive season. J. Dairy Sci. 97:6819-22.

Pérez J., Peña A.A., Valera D.L., Santamarina M.C. 2002. Proposal of a method of generation of layouts in milk goats livestock farms based in genetic algorithms. ASAE, St. Joseph, MI, USA, Paper No. 02-4021.

Pérez J., Santamarina M., Vallés J., Peña A., Valera, D., Carreño A. 2004. Optimal layout design for milk goats livestock farms using genetic algorithms. Agricultural Engineering International: the CIGR Journal of Scientific Research and Development. Vol.VI. Manuscript BC 03019.

Salimei E. 2011. Donkey. In: Encyclopedia of dairy sciences, 2nd ed. Elsevier Science \& Technology, Amsterdam, The Netherlands, 1:365-73.

Salimei E., Chiofalo B. 2006. Asses: milk yield and composition. In: N. Miraglia and W. Martin-Rosset (Eds.), Nutrition and feeding of the broodmare. EAAP Publication No 120. Wageningen Academic Publishers, Wageningen, The Netherlands, pp. 117-131.

Salimei E., Fantuz F. 2012. Equid milk for human consumption. Int. Dairy J. 24:130-42.

Shuai J. 2013. An augmented reality-based hybrid approach to facility layout planning. Ph. D. Diss., Nus Graduate School For Integrative Sciences and Engineering National University, Singapore.

Tomaselli G. Pappalardo G., Di Marco M. 2004. Building design solutions for sheep and goat breeding in the protected areas of Sicily. Agricultural Engineering International: the CIGR Journal of Scientific Research and development. Vol. VI. Manuscript BC 03026.

Wascher G., Merker J. 1997. A comparative evaluation of heuristics for the adjacency problems in facility layout planning. Int. J. Prod. Res. 35:447-66. 